\title{
Mercado del Cannabis medicinal en Colombia: una oportunidad para el sector salud que requiere lineamientos estratégicos del gobierno nacional y la academia
}

\author{
Mónica Ledezma-Morales* \\ Adriana Cristina Rodríguez** \\ Pedro Amariles***
}

\begin{abstract}
*Química Farmacéutica. Magister en Investigación y Uso Racional del Medicamento. Estudiante VII semestre de Doctorado en Ciencias Farmacéuticas y Alimentarias. Facultad de Ciencias Farmacéuticas y Alimentarias. Universidad de Antioquia. Grupo de Investigación Promoción y Prevención Farmacéutica. Medellín. Antioquia. Colombia.

**Química Farmacéutica. Magister en Epidemiología. Grupo de Investigación Promoción y Prevención Farmacéutica. Universidad de Antioquia. Medellín. Antioquia. Colombia.

***Químico Farmacéutico. Doctor en Farmacia (Farmacología). Grupo de Investigación Promoción y Prevención Farmacéutica. Universidad de Antioquia. Medellín. Antioquia. Colombia.

Correspondencia: Mónica Ledezma-Morales. Correo electrónico: monica.ledezma.morales@gmail.com
\end{abstract}

Resumen

Los resultados de las investigaciones sobre cannabis medicinal han traído a las mesas de gobierno discusiones sobre su potencial terapéutico y las medidas a tomar para controlar su producción, comercialización y utilización. En Colombia, desde el 2017, el mercado del cannabis medicinal se encuentra regulado; sin embargo, existen brechas entre la norma, disponibilidad y acceso de los pacientes a productos farmacéuticos con derivados del cannabis, aprobados por el ente regulatorio y que cumplan con los estándares de calidad. Desde esta perspectiva, se considera necesario que el gobierno nacional lidere, en coordinación con la academia y el sector productivo, el desarrollo de preparados farmacéuticos a base de cannabis; además de establecer e implementar medidas estratégicas y concertadas, orientadas a mejorar el acceso y la utilización adecuada de los productos farmacéuticos de cannabis medicinal, en especial para los casos en los que se pueda establecer una clara relación riesgo/beneficio. Con ello, se contribuye a mejorar las condiciones de salud de pacientes que se pueden beneficiar de estas opciones, al igual que al desarrollo y consolidación de un sector promisorio para el país. MÉD. UIS.2020;33(1):53-8.

Palabras clave: Marihuana Medicinal. Cannabinoides. Legislación de Medicamentos. Política de Salud.

\section{Medical Marijuana market in Colombia: an opportunity for the health sector that requires strategic guidelines from the national government}

Abstract

The results of medical marijuana research have brought to the government tables discussions about their therapeutic potential and the measures to be taken to control their production, commercialization and use. In Colombia, since 2017, the medical cannabis market is regulated; however, there are gaps between the laws, availability and the access of patients to pharmaceutical products with cannabis derivatives approved by the regulatory entity and that comply with the standards of quality. From this perspective, it is necessary for 
the national government to lead, in coordination with the academy and the productive sector, the development of pharmaceutical preparations based on cannabis and to establish and implement strategic and concerted measures, aimed to improve access and proper use of medical marijuana pharmaceutical products, mainly for cases in which a clear risk/benefit relationship can be established. This would help to improve the health conditions of patients who can get benefits from these options, as well as the development and consolidation of a promising sector for the country. MÉD.UIS.2020;33(1):53-8.

Keywords: Medical Marijuana. Cannabinoids. Health Policy.

¿Cómo citar este artículo?: Ledezma-Morales M, Rodríguez AC, Amariles P. Mercado del cannabis medicinal en Colombia: una oportunidad para el sector salud que requiere lineamientos estratégicos del gobierno nacional y la academia. MÉD.UIS.2020;33(1):53-8. doi: 10.18273/revmed. v33n1-2020006

La planta Cannabis sativa, también conocida como marihuana o cáñamo, ha sido utilizada desde la antigüedad por diversas culturas con fines medicinales, recreativos, agrícolas, cosméticos e incluso espirituales. Sin embargo, sólo hasta finales del siglo XX se comenzaron a diseñar y realizar estudios científicos orientados a evaluar sus efectos, a partir de allí, se identificó el Sistema Endocannabinoide y se dio inicio a la caracterización química y farmacológica de sus compuestos, denominados de forma genérica como "cannabinoides". Aunque se han identificado más de 100 de estos derivados, los más relevantes son el delta-9-tetrahidrocannabinol (THC) y el cannabidiol (CBD) por sus efectos en la salud dada su capacidad de ligarse e interactuar con 2 tipos de receptores de cannabinoides: los $C_{1}$ y $C_{2}$. De ellos, los $C_{1}$ se encuentran principalmente en el cerebro, los nervios periféricos y el sistema nervioso autónomo, mientras que los $\mathrm{CB}_{2}$ se localizan básicamente en neuronas y células del sistema inmune ${ }^{1,2}$. Por ello, atribuido a la distribución de los receptores y efectos de los cannabinoides, varios estudios han mostrado que el cannabis tiene beneficios en el manejo de diferentes patologías, posicionando el concepto de cannabis medicinal o terapéutico ${ }^{3-6}$.

Aunque, las autorizaciones para la comercialización y utilización terapéutica de estos productos podrían ser valoradas como conservadoras, las relacionadas con la investigación y utilización de la planta han facilitado el estudio de los efectos terapéuticos del cannabis $y$, con ello, la disponibilidad de un número creciente de publicaciones en los últimos años. En las mismas, se ha documentado actividad antiespasmódica, analgésica, antiemética, neuroprotectora, antiinflamatoria ${ }^{2}$ y ansiolítica apoyando la hipótesis del potencial terapéutico de los preparados farmacéuticos basados en cannabis medicinal, especialmente para enfermedades en las cuales las opciones terapéuticas son limitadas como la epilepsia refractaria ${ }^{6}$, la esclerosis múltiple, el síndrome de emaciación (caquexia) asociado al SIDA, el control del dolor en pacientes con cáncer y el manejo de náuseas asociadas a la quimioterapia ${ }^{2,7}$. Sin embargo, se han presentado limitaciones en la investigación y aprobación de productos farmacéuticos derivados del cannabis y, con ello, en su producción, comercialización y utilización; situación que podría atribuirse a tabús y restricciones normativas que han rodeado a la planta, dificultando así el acceso de los pacientes a estas opciones terapéuticas. Esto plantea la necesidad de establecer lineamientos estratégicos, gubernamentales y académicos, orientados a favorecer la generación, difusión y apropiación de evidencia de los resultados en salud alcanzados con la utilización de este tipo de productos, específicamente en condiciones en las cuales se puede establecer una clara relación riesgo/ beneficio.

En este contexto, se debe destacar que, el THC y el $\mathrm{CBD}$, generan efectos farmacológicos diferentes y a menudo contrarios ${ }^{8}$. Por ejemplo, al THC se le atribuyen la mayoría de los efectos psicológicos y físicos (psicoactivo); mientras que el CBD carece de psicoactividad y previene las respuestas psicóticas inducidas por THC en humanos ${ }^{1,8}$, por lo que se le considera clave como agente terapéutico. Es por esto que las concentraciones del THC determinan si los extractos de cannabis se consideran como psicoactivos o medicinales. De forma específica, en Colombia se ha definido que los extractos con concentraciones de THC superiores al $1 \%$ en peso seco, se valoran como cannabis psicoactivo; mientras los que tienen una concentración inferior al $1 \%$ de THC se valoran como no psicoactivo (medicinal) 9 . Sin embargo, se debe considerar que el efecto medicinal requiere, además del CBD, de la presencia del THC. 
Con respecto al efecto farmacológico de los cannabinoides en el manejo de las náuseas y vómitos asociados a quimioterapia, Einhorn y et al., realizaron un estudio doble ciego donde se reportó que el $75 \%$ de los pacientes tratados $(\mathrm{n}=80)$ preferían nabilona, un cannabinoide sintético, en comparación con la proclorperazina para el tratamiento de las náuseas y vómito durante sus ciclos de quimioterapia ${ }^{10}$. De igual manera, se han reportado efectos percibidos por los pacientes en enfermedades de difícil manejo como la esclerosis múltiple. Por ejemplo, Zajicek y et al., realizaron un ensayo aleatorizado, controlado con placebo, en el que trataron a 630 pacientes con esclerosis múltiple estable y espasticidad muscular ( 211 con extracto de cannabis oral, 206 con THC y 213 con placebo) durante 15 semanas. Como medida de efectividad se evaluó el cambio en los puntajes de espasticidad utilizando la escala de Ashworth, según la cual el efecto no fue estadísticamente significativo: Extracto vs placebo: 0,32 (IC95\%: -1,04 a 1,67); THC vs placebo 0,94 (IC95\%: -0,44 a 2,31) sin embargo, hubo evidencia del efecto sobre la espasticidad y el dolor informados por el paciente, con mejoras en la espasticidad informada del $61 \%$ para quienes fueron tratados con extracto de cannabis, $60 \%$ para aquellos con THC y del $46 \%$ para los pacientes que usaron placebo ${ }^{11}$.

Las propiedades terapéuticas de los cannabinoides también han sido objeto de estudio en la epilepsia refractaria, específicamente en niños y adultos jóvenes con síndrome de Lennox-Gastaut o sindrome de Dravet. En este sentido, Devinsky y et al., reportan un ensayo doble ciego controlado con placebo en donde se aleatorizaron 120 niños y adultos jóvenes para recibir tratamiento con CBD o placebo en adición al tratamiento epiléptico estándar, encontrando que la frecuencia media de convulsiones por mes disminuyó de 12,4 a 5,9 con CBD, en comparación con una disminución de 14,9 a 14,1 con placebo ${ }^{12}$.

En Estados Unidos, la Agencia de Medicamentos y Alimentos (FDA, por sus iniciales en inglés), con los debidos soportes científicos, ha autorizado la comercialización de medicamentos que contienen cannabinoides sintéticos, como es el caso del Cesamet ${ }^{\circledR}$ (nabilona/THC) y el Marinol ${ }^{\circledR}$ (dronabinol/ THC), indicados en el tratamiento de las náuseas y los vómitos inducidos por la quimioterapia ${ }^{13,14}$. Adicionalmente, el Marinol ${ }^{\circledR}$ está aprobado como estimulante del apetito en pacientes con SIDA ${ }^{14}$. De igual forma, el Epidiolex ${ }^{\circledR}$ (CBD), ha sido aprobado por la FDA y la Agencia Europea de Medicamentos
(EMA, por sus siglas en inglés), para el tratamiento de las convulsiones asociadas con el síndrome de Lennox-Gastaut o el síndrome de Dravet en pacientes desde los 2 años de edad'15,16. Así mismo, las agencias que regulan los medicamentos en Australia, Brasil, Canadá, España y Colombia (Instituto Nacional de Vigilancia de Medicamentos y Alimentos -INVIMA-) han autorizado la comercialización de un producto a base de extractos de la Cannabis Sativa, el Sativex ${ }^{\circledR}$ (THC/CBD) (en Brasil bajo el nombre comercial Mevatyl ${ }^{\circledR}$ ) indicado como tratamiento coadyuvante para la mejoría de los síntomas en pacientes adultos con espasticidad moderada o grave debida a la esclerosis múltiple y que no han respondido a otras terapias $^{17-20}$.

A pesar de la evidencia de los beneficios del cannabis medicinal en algunas afecciones, las implicaciones éticas, sociales y legales asociadas al cultivo, producción, manufactura, empaque, dispensación, uso, administración y reacciones adversas, generan controversias sobre la relación riesgo/beneficio del cannabis medicinal. Esta situación se podría mejorar, fundamentalmente, con dos acciones:

- Diseño y desarrollo de estudios que superen las limitaciones de las investigaciones disponibles, comolautilizacióndeuntratamientocomparador, tamaños de muestra adecuados, información detallada sobre la vía de administración, la concentración, composición y dosificación de los cannabinoides, al igual que procesos efectivos para identificar los posibles efectos adversos, incluyendo aquellos de largo plazo. En el caso de Colombia, esta situación se podría favorecer con la inclusión de más opciones de uso del cannabis en el listado de plantas medicinales aprobadas en el país, superando la única indicación de "antiinflamatorio de uso externo" considerando los problemas de salud y formas farmacéuticas de las que existe soporte de una relación riesgo/ beneficio favorable.

- Mejoramiento de la normatividad vigente para lograr claridad entre lo que se expide y el desarrollo del sector. En Colombia, por ejemplo, se expidieron en el 2017 el Decreto 613 y cinco resoluciones (579, 2891, 2892, 577 y 578), que regulan el cultivo, producción, importación, exportación, uso médico y científico del cannabis, entre otras. Sin embargo, no hay precisión sobre la distribución de los productos farmacéuticos que pueden contener derivados de cannabis 
(preparados magistrales, fitoterapéuticos o medicamentos), en qué tipo de pacientes y para qué tipo de patologías pueden ser prescritos, qué tipo de seguimiento y control se debe realizar a los pacientes que lo usen y en qué tipo de establecimientos farmacéuticos se pueden dispensar; aspectos que, en su conjunto, se ven como temas nucleares para la consolidación del sector productivo, pues con ello se tendría claridad sobre el círculo productivo completo.

En este marco, como debe ser y lo establece el Decreto 613 de 2017, los productos autorizados deben contar con registro sanitario otorgado por el INVIMA'; sin embargo, en Colombia sólo está aprobado un medicamento a base de cannabis, el Sativex ${ }^{\circledR}$, y aún no hay disponibles fitoterapéuticos con registro sanitario. Esto puede deberse a que la planta Cannabis sativa o indica, aunque aparece incluida dentro del Vademecum Colombiano de Plantas Medicinales ${ }^{21}$, solo está indicada para uso externo como analgésico.

Por ahora en Colombia el uso de preparados magistrales parece ser la única opción disponible que tienen los pacientes para acceder a medicamentos derivados del cannabis, los cuales deben ser elaborados bajo fórmulamédicay en establecimientos farmacéuticos certificados por el INVIMA en Buenas Prácticas de Elaboración y que estén inscritos en el Fondo Nacional de Estupefacientes ${ }^{9}$.

En términos del mercado esta situación se ve como una barrera para el acceso a medicamentos a base de cannabis medicinal, limitando el posible beneficio que este tipo de productos podría generar en algunos grupos de pacientes. Además, la falta de medicamentos y fitoterapéuticos autorizados, sumado al elevado costo de los preparados magistrales podrían favorecer la utilización de productos de dudosa procedencia, elaborados en lugares sin certificación en Buenas Prácticas de Elaboración, generando dudas en la calidad fisicoquímica y microbiológica del material vegetal empleado, de los procesos de fabricación y de las concentraciones de CBD y THC de los preparados, lo que se convierte en un riesgo para la salud de los posibles usuarios.

Aunque el uso de aceites, vaporizadores, ungüentos, etc., no está soportado por evidencia científica, los pacientes lo continuan utilizando como un último recurso, por lo que la discusión en materia de cannabis medicinal debe incluir las estrategias a implementar por el gobierno y los profesionales de la salud para garantizar productos de calidad y un uso adecuado.

En este sentido, el Departamento de Salud del gobierno australiano publicó en el 2017 una guía para el uso y administración de cannabis medicinal para la prevención de náuseas y vómito ${ }^{22}$. En dicha guía, acorde con la información disponible, se mencionan varias limitaciones como el alto riesgo de sesgo de los ensayos clínicos y la poca o nula información sobre el uso de estos preparados en embarazo, pacientes pediátricos, adultos mayores, y personas con insuficiencia hepática o renal. Sin embargo, la guía presenta de forma clara los pros y los contras de su utilización, los estados de salud para los cuales es eficaz, las recomendaciones con respecto a las dosis, las reacciones adversas más frecuentes con el uso de cannabis medicinal y las indicaciones de cuándo terminar la terapia. La guía establece que los productos de cannabis medicinal pueden ser efectivos para la náusea y el vómito pero sólo deben ser prescritos cuando los tratamientos estándar hayan fallado y deben usarse como coadyuvante de la terapia farmacológica para estabilizar y mejorar la calidad de vida de los pacientes ${ }^{22}$.

Por su parte, la Sociedad Estadounidense de Farmacéuticos también desarrolló una guía de asesoría integral basada en la evidencia del uso de medicamentos naturales a base de cannabis usados por vía oral para tratamiento del dolor crónico ${ }^{23}$. Estas guías constituyen instrumentos importantes para farmacéuticos y profesionales de la salud dado que proporcionan información sobre cómo aconsejar a los pacientes de manera efectiva sobre su uso. Aunque aún está pendiente la evaluación de la utilidad de estas guías en el manejo de los pacientes, las mismas pueden considerarse como referencia en Colombia para el uso de derivados del cannabis en las patologías ya estudiadas.

Desde esta perspectiva se considera necesario que el gobierno nacional lidere en coordinación con la academia y el sector productivo, el desarrollo de preparados farmacéuticos a base de cannabis. Esto podría ayudar a mejorar la calidad de vida de los pacientes y quizás, reducir los sobrecostos (por aumento de medicamentos prescritos, consultas reiteradas a especialistas y estancias hospitalarias prolongadas) asociados al poco control de enfermedades como el Síndrome de Lennox- 
Gastaut, para el que ya está aprobado el uso de estos medicamentos. Adicionalmente, se deben establecer lineamientos para la prescripción y seguimiento de los pacientes que usan cannabis medicinal. Esta estrategia se debe acompañar del seguimiento $y$ registro de los resultados de efectividad y seguridad alcanzados en los pacientes que lo usan bajo prescripción médica, con la especificación del producto empleado.

En este contexto, el INVIMA publicó una circular relacionada con las condiciones/requisitos para la certificación de las Buenas Prácticas de Elaboración ${ }^{24}$ y, más recientemente, el Ministerio de Salud y Protección Social expidió la Resolución 315 de 2020, en la cual se precisan las características que determinan a algunos productos farmacéuticos elaborados a partir de cannabis, en medicamentos de control especial y fiscalizados. Indicando que son de control especial los medicamentos de síntesis química, fitoterapéuticos y las preparaciones magistrales, que contengan una cantidad igual o superior a $2 \mathrm{mg}$ de THC por tableta, cápsulas o similar, o por cada gramo o mililitro en caso de soluciones, cremas y similares ${ }^{25}$, lo que define las condiciones de prescripción y dispensación de estos productos.

Aunque dichos documentos representan avances que pueden facilitar la prescripción de preparados magistrales en ciertos pacientes y con ello aumentar la evidencia clínica de los resultados en salud, se requiere de más alternativas. Por ello, es clara la necesidad de acompañar la legislación que regula los componentes o materias primas (productos intermedios) con normas, estrategias y guías que favorezcan el desarrollo y utilización de productos finales, como lo han hecho países como Australia ${ }^{22}$, Estados Unidos ${ }^{23}$ y más reciente Brasil $^{26}$ con la estrategia de Registros Sanitarios Abreviados, en el contexto de productos a base de cannabis y no de medicamentos en el sentido estricto, lo que puede generar beneficios para el sistema de salud y los pacientes.

Las condiciones de nuestro país nos demandan establecer e implementar medidas estratégicas y concertadas orientadas a mejorar el acceso y utilización adecuada de los productos farmacéuticos derivados de cannabis medicinal, para los casos en los que se pueda establecer una clara relación riesgo/beneficio. Con ello, se contribuye a mejorar las condiciones de salud de pacientes que se pueden beneficiar de estas opciones, al igual que al desarrollo y consolidación de un sector promisorio para el país.

\section{Conflicto de intereses}

Los autores declaran no tener ningún conflicto de intereses.

\section{Referencias bibliográficas}

1. Pacher P, Kogan NM, Mechoulam R. Beyond THC and Endocannabinoids. Annu Rev Pharmacol Toxicol. 2020;60:637-659.

2. Pertwee RG. Emerging strategies for exploiting cannabinoid receptor agonists as medicines. Br J Pharmacol. 2009;156(3):397411.

3. Bridgeman MB, Abazia DT. Medicinal Cannabis: History, Pharmacology, And Implications for the Acute Care Setting. P T Peer-Rev J Formul Manag. 2017;42(3):180-8.

4. Resstel LBM, Tavares RF, Lisboa SFS, Joca SRL, Corrêa FMA, Guimarães FS. 5-HT1A receptors are involved in the cannabidiolinduced attenuation of behavioural and cardiovascular responses to acute restraint stress in rats. Br J Pharmacol. 2009;156(1):181-8.

5. Schier AR de M, Ribeiro NP de O, Silva AC de O e, Hallak JEC, Crippa JAS, Nardi AE, et al. Cannabidiol, a Cannabis sativa constituent, as an anxiolytic drug. Rev Bras Psiquiatr Sao Paulo Braz 1999. 2012;34 Suppl 1:S104-110.

6. O’Connell BK, Gloss D, Devinsky O. Cannabinoids in treatmentresistant epilepsy: A review. Epilepsy Behav EB. 2017;70(Pt B):341-8.

7. Whiting PF, Wolff RF, Deshpande S, Di Nisio M, Duffy S, Hernandez AV, et al. Cannabinoids for Medical Use: A Systematic Review and Meta-analysis. JAMA. 2015;313(24):2456-73.

8. Martin-Santos R, A. Crippa J, Batalla A, Bhattacharyya S, Atakan Z, Borgwardt S, et al. Acute Effects of a Single, Oral dose of d9-tetrahydrocannabinol (THC) and Cannabidiol (CBD) Administration in Healthy Volunteers. Curr Pharm Des. 2012;18(32):4966-79.

9. Ministerio de Salud y Protección Social. Decreto 613 de 2017. oct 4, 2017.

10. Einhorn LH, Nagy C, Furnas B, Williams SD. Nabilone: an effective antiemetic in patients receiving cancer chemotherapy. J Clin Pharmacol. septiembre de 1981;21(S1):64S-69S.

11. Zajicek J, Fox P, Sanders H, Wright D, Vickery J, Nunn A, et al. Cannabinoids for treatment of spasticity and other symptoms related to multiple sclerosis (CAMS study): multicentre randomised placebo-controlled trial. Lancet Lond Engl. el 8 de noviembre de 2003;362(9395):1517-26.

12. Devinsky O, Cross JH, Laux L, Marsh E, Miller I, Nabbout R, et al. Trial of Cannabidiol for Drug-Resistant Seizures in the Dravet Syndrome. N Engl J Med. el 25 de mayo de 2017;376(21):2011-20.

13. Food and Drug Administration. Cesamet Label. [Internet]. 2006. Disponible en: https://www.accessdata.fda.gov/drugsatfda_docs/ label/2006/018677s011lbl.pdf

14. Food and Drug Administration. Marinol. Highlights of prescribing information. [Internet]. 2017. Disponible en: https://www. accessdata.fda.gov/drugsatfda_docs/label/2017/018651s029lbl.pdf

15. Food and Drug Administration. Epidiolex. Highlights of prescribing information. [Internet]. 2018. Disponible en: https:// www.accessdata.fda.gov/drugsatfda_docs/label/2018/210365lbl. pdf

16. European Medicines Agency. Epidyolex [Internet]. European Medicines Agency. 2019 [citado el 25 de febrero de 2020]. Disponible en: https://www.ema.europa.eu/en/medicines/human/ EPAR/epidyolex

17. Agencia Española de Medicamentos y Productos Sanitarios. SATIVEX [Internet]. [citado el 25 de febrero de 2020]. Disponible en: https:/cima.aemps.es/cima/publico/detalle. html?nregistro $=72544$

18. Health Canada. Fact Sheet SATIVEX [Internet]. 2005 [citado el 
25 de febrero de 2020]. Disponible en: https://www.canada.ca/ en/health-canada/services/drugs-health-products/drug-products/ notice-compliance/conditions/fact-sheet-sativex.html

19. Agencia Nacional de Vigilancia Sanitaria-Brasil. Mevatil [Internet]. [citado el 25 de febrero de 2020]. Disponible en: https://consultas.anvisa.gov.br/\#/medicamentos/2535173807420 1441/?nomeProduto=Mevatyl

20. Instituto Nacional de Vigilancia de Medicamentos y Alimentos. Consulta Registros Sanitarios [Internet]. [citado el 23 de octubre de 2019]. Disponible en: http://consultaregistro.invima.gov. co:8082/Consultas/consultas/consreg encabcum.jsp

21. Instituto Nacional de Vigilancia de Medicamentos y Alimentos. Listado de plantas medicinales [Internet]. 2019 [citado el 23 de octubre de 2019]. Disponible en: https://paginaweb.invima.gov. co/normatividad/normatividad-interna/proyectos.html

22. Australian Government Department of Health Therapeutic Goods. Guidance for the use of medicinal cannabis for the prevention or management of nausea and vomiting in Australia [Internet] 2017 [citado el 23 de octubre de 2019]. Disponible en: https:// www.tga.gov.au/publication/guidance-use-medicinal-cannabisprevention-or-management-nausea-and-vomiting-australia

23. Makary P, Parmar JR, Mims N, Khanfar NM, Freeman RA. Patient Counseling Guidelines for the Use of Cannabis for the Treatment of Chemotherapy-Induced Nausea/Vomiting and Chronic Pain. J Pain Palliat Care Pharmacother. 2018;32(4):216-25.

24. Instituto Nacional de Vigilancia de Medicamentos y Alimentos. Guía para las visitas de buenas prácticas de elaboración de preparaciones magistrales a base de cannabis [Internet]. 2020 [citado el 30 de enero de 2020]. Disponible en: https://www. invima.gov.co/documents/20143/908412/guia-para-las-visitas-debuenas-practicas-de-elaboracion-de-preparaciones-magistralesa-base-de-cannabis ASS-AYC-GU017.pdf/8021bdd8-13f1-4cf6fe69-39163ee09eef? $\bar{t}=1572042596864$

25. Ministerio de Salud y Protección Social. Resolución 315 de 2020. mar 2, 2020.

26. Ministério da Saúde/Agência Nacional de Vigilância Sanitária/ Diretoria Colegiada. Resolução da diretoria colegiada - RDC N ${ }^{\circ}$ 327, de 9 de dezembro de 2019. Dispõe sobre os procedimentos para a concessão da Autorização Sanitária para a fabricação e a importação, bem como estabelece requisitos para a comercialização, prescrição, a dispensação, o monitoramento e a fiscalização de produtos de Cannabis para fins medicinais, e dá outras providências. [Internet]. 2019 [citado el 30 de enero de 2020]. Disponible en: http:/www.in.gov.br/en/web/dou/-/ resolucao-da-diretoria-colegiada-rdc-n-327-de-9-de-dezembrode-2019-232669072 